\title{
Reliability Measurement of Corneal Wavefront Aberrations using Corneal Videokeratograph Atlas 9000
}

Noorshazana Mat Rejab @ Md Rejab¹, Mohd Radzi Hilmi ${ }^{1}$, Khairidzan Mohd Kamal², Md Muziman Syah Md Mustafa ${ }^{1}$

${ }^{1}$ Department of Optometry And Visual Science, International Islamic University Malaysia, Pahang, Malaysia

2Department of Ophthalmology, International Islamic University Malaysia, Kuantan, Pahang

Introduction: Accurate corneal wavefront aberration measurements are essential in determining patient's suitability, vision outcomes and patient satisfaction in laser refractive surgeries. This study aimed to evaluate the reliability measurement of higher-order aberration (HOA) using corneal videokeratograph Atlas 9000 . Materials and method: 38 eyes of 19 participants were recruited in this study. Comprehensive eye examination was done to ensure all participants eligibility, and any conditions in which obstruct the central cornea were excluded. Corneal wavefront aberrations were measured based on Zernike polynomials. In assessing repeatability, three repetitive measurements in five minutes in a single session were taken by 2 experience examiners. For reproducibility measurement, similar approach was done with the time-interval between measurements was set at one week. Bland-Altman, limits of agreement (LoA) and intraclass correlation coefficients (ICCs) were used to evaluate the reliability measurement. Results: Bland-Altman and LoA findings revealed no significant differences for both repeatability and reproducibility measurement. For repeatability testing, the mean differences for vertical trefoil, oblique trefoil and spherical aberration were $-0.096 \pm 0.493,0.001 \pm 0.048,0.008 \pm$ $0.035,0.004 \pm 0.029,0.010 \pm 0.053$ with LoA of $1.930,0.188,0.138,0.114$, and 0.208 respectively. Intraclass correlation coefficient (ICC) excellent reliability of 0.841 for all parameters. Likewise, reproducibility testing showed similar findings with the mean difference were $-0.018 \pm 0.091,0.016 \pm 0.061,-0.0004 \pm 0.036,-0.002 \pm 0.042$, $0.003 \pm 0.026$, with LoA of $0.356,0.24,0.141,0.164$, and 0.102 respectively. Intraclass correlation coefficient (ICC) shows excellent reliability of more than 0.9 for all parameters. LoA of less than 1.0 were observed in all measurements (except for repeatability of vertical trefoil) indicates high consistency of the measurements. Conclusions: Corneal videokeratograph Atlas 9000 provides excellent HOA measurement reliability. 